\title{
Black carp growth hormone gene transgenic allotetraploid hybrids of Carassius auratus red var. (우)×Cyprinus carpio $(ð)$
}

\author{
FENG $\mathrm{Hao}^{\dagger}$, FU YongMing ${ }^{\dagger}$, LUO Jian, WU Hui, LIU Yun \& LIU ShaoJun* \\ Key Laboratory of Protein Chemistry and Developmental Biology of Ministry of Education of China, College of Life Sciences, \\ Hunan Normal University, Changsha 410081, China
}

Received December 6, 2010; accepted March 4, 2011; published online August 2, 2011

\begin{abstract}
Ecological safety is a major consideration in the commercialization of transgenic fish. Development of sterile transgenic triploid fish through hybridization of transgenic tetraploid fish and transgenic diploid fish is a feasible way to solve this problem. The "all-fish" transgene, $p b c A b c G H c$, containing the black carp $\beta$-actin gene promoter and the open reading frame (ORF) of the black carp growth hormone $(\mathrm{GH})$ gene was constructed and introduced into fertilized eggs of allotetraploid fish through microinjection. Contrast cultivation results showed that the growth rate of 150 day-old $\mathrm{P}_{0}$ black carp GH gene transgenic allotetraploid fish was much higher than that of controls. Sixty 150 day-old transgenic allotetraploid fish were assayed by PCR for transgene integration and $90 \%$ of fish were positive for the transgene. The transgene was detected in 13 of 20 sperm samples from male transgenic allotetraploid fish. RT-PCR detected transcription of the exogenous black carp GH gene in the muscle, liver, kidney and ovaries of the largest transgenic allotetraploid fish. This study has developed $\mathrm{P}_{0}$ black carp GH gene transgenic allotetraploid fish with a highly increased growth rate, which provides a solid foundation for the establishment of a pure line of transgenic allotetraploid fish and for the large scale production of sterile transgenic triploid fish.
\end{abstract}

transgene, allotetraploid fish, growth hormone, black carp, ecological safety

Citation: $\quad$ Feng H, Fu Y M, Luo J, et al. Black carp growth hormone gene transgenic allotetraploid hybrids of Carassius auratus red var. ( 우) $\times$ Cyprinus carpio (đ). Sci China Life Sci, 2011, 54: 822-827, doi: 10.1007/s11427-011-4210-x

Genetic engineering has been widely applied in breeding studies since transgenic mice with outstanding growth rate were developed by Palmiter in 1982. Today, transgenesis is one of the most popular and effective biological tools in life sciences. Since the first transgenic fish was developed by a Chinese scientist in 1985 [1], fish transgenesis has been applied to many fields including fish production, ecology and comparative biology. Transgenic fish with desirable features derived from the transgene have been developed through gene transfer and genetic selection, which is beyond the scope of traditional breeding [2-8].

Food safety and ecological concerns are major issues for the production of transgenic fish. No significant differences

$\dagger$ Contributed equally to this work

*Corresponding author (email: 1sj@ hunnu.edu.cn) were observed in physiological assays between animals fed on GH gene transgenic fish and animals fed on a control diet, which implied that characterizing the food safety of transgenic fish needs further study [9]. However, frequent accidents resulting in the escape of transgenic fish suggested that the ecological risks posed by transgenic fish are of paramount importance [10]. If transgenic fish escape into the environment, they are likely to become the dominant species because of stronger viability and competitiveness derived from the transgenes. This may result in them overwhelming ecological niches of other wild fish, leading to altered fish populations and changes in species diversity. In addition, transgenic fish may hybridize with close relatives and affect genetic diversity [11]. The fry of growth hormone (GH) gene transgenic fish were more susceptible to being 
preyed upon than controls in a simulated natural ecosystem $[12,13]$. However, GH gene transgenic fish showed advantages with respect to migration ability, growth rate and resistance against diseases if there was no natural enemy in the environment [14-16]. Thus, the ecological safety of transgenic fish is of primary importance if they are to be used for commercial fish production [17].

Sterile transgenic fish do not reproduce; therefore, they do no harm to the species or genetic diversity of a natural ecosystem. Thus, sterile transgenic fish is a possible answer to the ecological problem of transgenic fish, and the development of triploid transgenic fish is an effective way to obtain sterile transgenic fish. Artificially induced fish were not all triploid or sterile when physical or chemical methods were recruited. However, the production of triploid hybrids from transgenic tetraploid fish and transgenic diploid fish may be a feasible and effective solution for this issue. Allotetraploid fish had been developed through the cultivation and genetic selection of tetraploid hybrids of the red crucian carp (Carassius auratus red var. (우)) and the Xiangjiang River carp (Cyprinus carpio (ठ)). These allotetraploid fish have established a new fish population after 20 years of reproduction, in which both males and females are fertile [18]. These allotetraploid fish could be used as the parental strain for triploid fish production and they provide a good opportunity for the commercial production of transgenic fish. Triploid hybrids have been produced on a large scale by breeding allotetraploid fish with regular diploid crucian carp or common carp and now occupy a certain market share in China. These fish are completely sterile, as verified by biological assays and years of culturing [19].

In this paper, the "all-fish" transgene, which is composed of the black carp $\beta$-actin gene promoter and the ORF of the black carp GH gene, was constructed and introduced into the eggs of allotetraploid fish. Contrast cultivation demonstrated that $\mathrm{P}_{0}$ black carp $\mathrm{GH}$ transgenic allotetraploid had a higher growth rate than control fish. This provides a solid foundation for the establishment of a black carp GH transgenic allotetraploid fish line.

\section{Materials and methods}

\subsection{Materials}

Black carp, gynogenetic grass carp and allotetraploid fish were obtained from the tetraploid fish protection base at Hunan Normal University. Reagents were purchased as follows: EX Taq DNA polymerase from Takara (Dalian, China), pUCM-T vector for T/A cloning, gel extraction kit and PCR purification kit from Sangon, restriction endonuclease from New England Biolabs (Ipswich, MA, USA) and the first strand cDNA synthesis kit from Promega (Madison, Wisconsin, USA). DNA sequencing was performed by Sangon (Shanghai, China).

\subsection{Construction of an "all-fish" transgene}

pUCM-bcA, which contains the black carp $\beta$-actin gene promoter, and pbcGHc, which contains the black carp GH gene ORF were previously prepared by our laboratory [20,21]. The 3' UTR of the GH gene from gynogenetic grass carp was PCR amplified with the primers, $P_{5}$ (TGTCGCTGGATCCAKGGCRCCRRTGTATT) and $\mathrm{P}_{3}$ (TGTCGCTCTCGAGCAGATCCTGGTCATTT). BamH I and Xho I sites are indicated by shaded text. Genomic DNA was isolated from the fin of gynogenetic grass carp and used as template. The 3' UTR from the GH gene of gynogenetic grass carp was cloned into pUCM and the construct was named pUCM-GC3'. The primers, $\mathrm{P}_{\mathrm{f}}$ and $\mathrm{P}_{(\mathrm{dq}-)}$, were used to amplify the promoter of the black carp $\beta$-actin gene. $\mathrm{P}_{(\mathrm{dq}+)}$ and $\mathrm{P}_{\mathrm{r}}$ were used to amplify the ORF of the black carp GH gene. Ligation PCR, using $\mathrm{P}_{\mathrm{f}}$ and $\mathrm{P}_{\mathrm{r}}$, was performed to link the two fragments. The amplified fragment was cloned into pUCM-T and the construct was named pUCM-A/GH. The 3' UTR of the GH gene from gynogenetic grass carp was excised from pUCM-GC3' and cloned into pUCM-A/GH immediately downstream of the black carp GH gene ORF, between BamH I and Xho I sites. The "all-fish" transgene was named pbcAbcGHc. The primers used were $P_{f}$ (ATGAATTCGTTTGATSAAAATCGCYT); $\mathrm{P}_{(\mathrm{dq}+)}$ (ACAGTTCAGCCATGGCTAGAGCRTTAGTGCT); $\mathrm{P}_{\left(\mathrm{dq}_{-}\right)}$(AGCACTAAYGCTCTAGCCATGGCTGAACTGT); $\mathrm{P}_{\mathrm{r}}$ (TGTCGCTGGATCCTTACAGGGTGCAGTTGGAAT). EcoR I, Nco I and BamH I sites are indicated by shaded text.

\subsection{Gene transfer and contrast cultivation}

The pbcAbcGHc plasmid was digested with EcoR I and $X h o$ I and the purified transgene $(2.9 \mathrm{~kb})$ was dissolved in ST solution ( $10 \mathrm{mmol} \mathrm{L}^{-1}$ Tris $\mathrm{Cl}, 100 \mathrm{mmol} \mathrm{L}^{-1} \mathrm{NaCl}$ ) at $100 \mathrm{ng} \mu \mathrm{L}^{-1}$. The DNA (1-2 $\left.\mathrm{nL}\right)$ was microinjected into each fertilized allotetraploid fish egg before the first cleavage. Contrast cultivation was performed in the tetraploid fish protection base at Hunan Normal University as previously described [22].

\subsection{Characterization of the transgenic fish}

Eighty-seven $\mathrm{P}_{0}$ transgenic allotetraploid fish and 100 control fish were chosen randomly and used when they were 150 days old.

\subsubsection{Weight and body length}

The weight and body length of all fish were measured as described previously [22].

\subsubsection{Integration of the transgene}

Genomic DNA was isolated from the caudal fin of 60 transgenic allotetraploid fish and from 20 controls. Genomic DNA was also prepared from the sperm of 20 male trans- 
genic allotetraploid fish. $\mathrm{P}_{1}$ and $\mathrm{P}_{2}$ primers were used for PCR amplification of the integrated transgene. Amplified DNA fragments were chosen arbitrarily for sequencing: $P_{1}$ (TGGCGTGATGAATGTCG) and $\mathrm{P}_{2}$ (AACACGGATGACTGCGT).

\subsubsection{Transcription of the transgene}

The largest transgenic allotetraploid fish (female, $700 \mathrm{~g}$ ) and the largest control (female, $195 \mathrm{~g}$ ) were chosen to investigate transgene transcription. Total RNA was isolated separately from the caudal fin, muscle, liver, kidney, spleen, intestine, heart and ovary and subsequently used for RT-PCR with the primers $P_{a}$ and $P_{b}$. All the RT-PCR amplified fragments were sequenced: $P_{a}$ (ATGGCTAGAGCRTTAGTGCTGTT); $P_{\mathrm{b}}$ (TTACAGGGTGCAGTTGGAATCC).

\section{Results}

\subsection{Construction of the "all-fish" transgene}

The promoter of the black carp $\beta$-actin gene and the ORF of the black carp GH gene were precisely fused in pUCMA/GH, which was verified by Nco I digestion (data not shown) and DNA sequencing. Two nucleotides in the promoter were mutated during cloning (A312 was altered to $\mathrm{G}$ and T934 to C, following alignment with AY289135) and there were two nonsense mutation sites in the GH gene (C10 to A and G385 to A, following alignment with AF389238). The amplified 3' UTR fragment of the gynogenetic grass carp GH gene was 660 bp and had $98.7 \%$ identity to that of grass carp (X60988 or X60419). The "all-fish" transgene vector, $p b c A b c G H c$, was constructed after the 3' UTR of the gynogenetic grass carp GH gene had been cloned into pUCM-A/GH, between BamH I and Xho I sites, in which the ORF of the black carp GH gene had been fused into the position of the first exon of the black carp $\beta$-actin gene. The $p b c A b c G H c$ transgene was approximately $2.9 \mathrm{~kb}$ and its sequence and structure are shown in Figure 1.

\subsection{Weight and body length of the transgenic allotet- raploid fish}

The weight and body length of 150 day-old transgenic allotetraploid fish were measured as described previously [22]. The average weight of 87 transgenic allotetraploid fish was $296.3 \mathrm{~g}$ and the average body length was $21.7 \mathrm{~cm}$. The largest transgenic allotetraploid fish (female) weighed $700 \mathrm{~g}$. The average weight of 100 controls was $104.2 \mathrm{~g}$ and the average body length was $16.1 \mathrm{~cm}$. The largest control (female) weighed $195 \mathrm{~g}$. The average weight of transgenic tetraploid fish was 2.8 times that of control fish and the average body length of the transgenic fish was 1.3 times that of the control fish. The largest transgenic allotetraploid fish was 3.6 times that of the largest control fish. Black carp GH gene transgenic allotetraploid fish had a higher growth rate than the controls (Figure 2 and Table 1) and some transgenic fish with outstanding growth rates showed a protuberance behind the head because of thick muscles on the back (Figure 3). Sperm could be pressed out from 32 male transgenic fish; however, there was no such phenotype among the 150 day-old controls.

\subsection{Integration and transcription of the transgene}

PCR with primers $P_{1} / P_{2}$ was performed to detect the exogenous transgene and the predicted amplified band was 411 bp. The transgene was detected in 54 caudal fins of 60 transgenic allotetraploid fish but not in any control fish, which suggested the integration of the transgene in the caudal fin was $90 \%$. Moreover, the transgene was detected in each of the 20 male transgenic fish. The transgene was detected in 13 sperm samples from these 20 male transgenic fish, which indicated that the transgene could be passed to the next generation. Representative PCR results are shown in Figure 4. Amplified transgene fragments from eight fish were randomly chosen and sequenced. Each fragment had a $98 \%$ or higher identity to $p b c A b c G H c$ and the Nco I site was found in each sample.

Total RNA was isolated from caudal fin, muscle, liver, kidney, spleen, intestine, heart and ovary of the biggest transgenic fish $(700 \mathrm{~g})$ and from the biggest control fish (195 g). RT-PCR, using primers $\mathrm{P}_{\mathrm{a}}$ and $\mathrm{P}_{\mathrm{b}}$, was used to detect the transcription of $p b c A b c G H c$ in the transgenic fish. The predicted amplified fragment ( $630 \mathrm{bp}$ ) was found in the muscle, liver, kidney and ovary of the transgenic fish (Figure 5) but not in control tissues (data not shown). The amplified fragments from the four tissues were sequenced and all showed $98 \%$ or higher identity to the ORF of the black carp GH gene. A Sac I site, in the ORF of the black carp $\mathrm{GH}$ gene, was found in each of the four fragments.

\section{Discussion}

Ecological safety is a major factor in the production of transgenic fish; however, this can be addressed by the development of sterile triploid transgenic fish. Triploid transgenic fish cannot form a distinct species and therefore have no impact on the genetic diversity of natural ecosystems. Development of triploid transgenic fish through breeding transgenic tetraploid fish with transgenic diploid fish is a feasible way to produce sterile transgenic fish on a large scale. However, generating tetraploid fish is the most difficult step of this strategy. Over the past decade, Hunan Normal University has successfully developed an allotetraploid fish species through the cultivation and genetic selection of allotetraploid hybrids of red crucian carp (Carassius auratus red var. 우) $\times$ Xiangjiang River Carp (Cyprinus carpio $\widehat{\jmath}^{\Uparrow}$ ). Sterile triploid hybrids had been successfully pro- 
A

ttgatgaaaatcgcttaggccttgttcttcaactagtctagcttccccttcttcactctcaagttgcaagaaagcaagtgtagcaatgtgcacgcgacagecgggtgtgtgacgctggaCCA ATcagagggcagagetccgaaagtttacctttatggetagagecgggcatatgccgtca TATAaaagagctcgcccagctttcaacctcacttgagctcctccacacgcagctagtg cggaatatcatcagettgtaacccattctcttaagtcgacaaaccccccaaacctaaggtgagttgattttcagetttattgcattttagttcattaatattaattaaacctgtaactatgatgtata aataaaactggatgaggaaattagattaagttaccggtctcttcgetttaagttctaacttctgettaaaaacggtagttgtttaatatatagttattttatgtaaatgttgctgtattattataactttat gtacttactgggcatgtcaggtggaaacgacggtatccgttgtaggcacgacattaaatgggecggtgtgaaataagtgttgcctttcttaacattaagatgtgetctgattaaccgtgctttaa caactataacttgacttgacagtttaagggtattatttgaggcatgttacacacttgatggatagccggcatgggaagttcttgtgcaggcagtgctgcagaagggtgtgacctactttagct agccggctaaccagegttcacettctgtaaacctggtgaacctaattcacttattcgaacactaaattatcaaagattggttattagttcactttcaacacacccaacattttagtgaatactggege tttataatgtagtatgcagcatgcagccettcagtctttcttaaaactgaggattagtcaagtattcattcactattaaagcgttcgaaatataaggtgtggtcaatttaataactggggtaaagtg ttccggttgcatttaaactttataattgtgtcgaacagtaattttcttaaaactaattgataaagactagaatataaactgaatgggctgatggtgatctctcacaagtgettcggcgtttagtatgct cttatggagtcattgaagtgactgcagatctgtgacgcagtaatgttgggcagacaccegtcgaaattcggttgtgtaattgataccaggegaggatgaaagaggatgtaaaacttcattcgtg tagaatttagggagtggecetggegtgatgaatgtcgaaatetgttcetttttactgaaccatacgactetggetgagtgecacaccgecggcagecgcaaageggetcaatccattgecttt atggtaataatgggagaatgcagagggacttccttgtctggcatatctgaggegegcattgtcactctagcacccactagcggttagactgcagaatgcagcatgaaacaggaagttgactc cacatggtcacatgctcactggcactttcttacattgcagcagtgcacttcaaaacactttctctctctttacagttcagec ATGgctagagegttagtgetgttgtcggtggtgctggttagt ttgttggtgaaccaggggacggcctcagagaaccagcggctcttcaacaacgcagtcatccgtgttcaacacctgcaccagctggctgcaaaaatgattaacgacttcgaggacaacctgtt gcctgaggaacgcagacagctgagtaaaatctttcctctgtcttctgcaactctgactctattgaggcgcccactggaaaagatgaaacacagaagagctctatgttgaagctccttcgcatct cttccgectcattgagtcctgggagttccccagccaaaccctgagcggagccatctcaaacagcctgactgtcgggaaccccaaccagatcactgagaagetggctgacttgaaagtggg catcagcgtgctcatcaagggatgtctggatggtcaaccaaacatggatgataacgaatccctgccgctgcctttgaggatttctacttgaccatgggggagagcagcctcagagagagctt tcgtcttcttgcttgettcaagaaggacatgcacaaggtggaaacttacctgagggttgcaaattgcaggagatccetggattccaactgcaccetgTAA ggatccagggegccgatgtatt gtagccaaagcctgtgacacactttgctgcaaatctaaaaccagtttaagtcctcaaaatctectaatataattattatctggtcttatatatgcaggaaatgtcaaccaggcatggetaggtctgtt ctctagttccctcccatatctaaacccaacactattgtattattcttctcattggggagtgetcgtaaattaaagacattaagatctgatttaacattcacagtggtgctaagcaatatatggcaatat atttcaaatgtgeccaaatcgettgactctagtatttatggctccaaaatggetaaagatgectttgtcgaaactgtcatttggatggatgggttcactcccaaccaagtgtatgaatgtaaac attgtctgtctgatagattatgtccatattattagetcatgetgttctcttgaagetgtgtgtcttatccattaaattctaaactgcatccaatgtgtetgctgtatgacetgtgtggttatttttctacc cgtctcaacattacacgtaccttacatttcactgctcggatattcatcaaaatcagtcctatcatccgtccacaatgaacgagttcaaaatggaccgaaaaaaagaccaggatctg

B

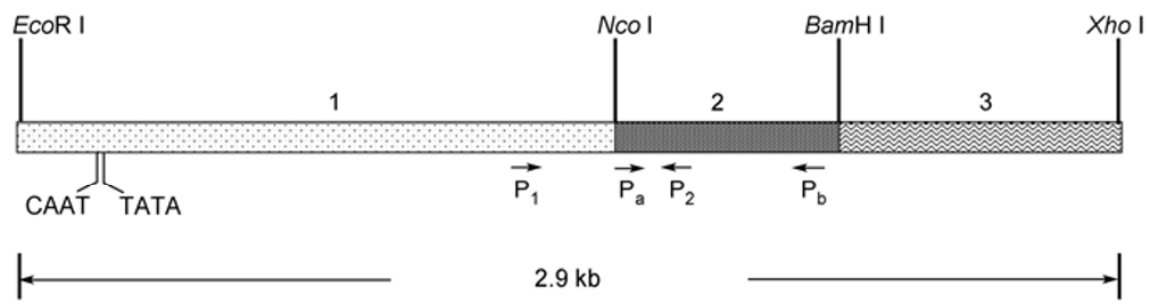

Figure 1 "All-fish" transplant gene $p b c A b c G H c$. A, The sequence of the $p b c A b c G H c$ transgene. The black carp GH ORF is shown in the frame. CCAAT and TATA boxes are capitalized. $N c o$ I and Bam H I sites are shown in shaded type. B, 1, $5^{\prime}$ flanking sequence of the black carp $\beta$-actin gene; 2 , ORF of the black carp GH gene; 3, 3' UTR of the GH gene from gynogenetic grass carp; $\mathrm{P}_{1} / \mathrm{P}_{2}$ and $\mathrm{P}_{\mathrm{a}} / \mathrm{P}_{\mathrm{b}}$ represent the PCR primers for detecting integration and transcription of the transgene, respectively.
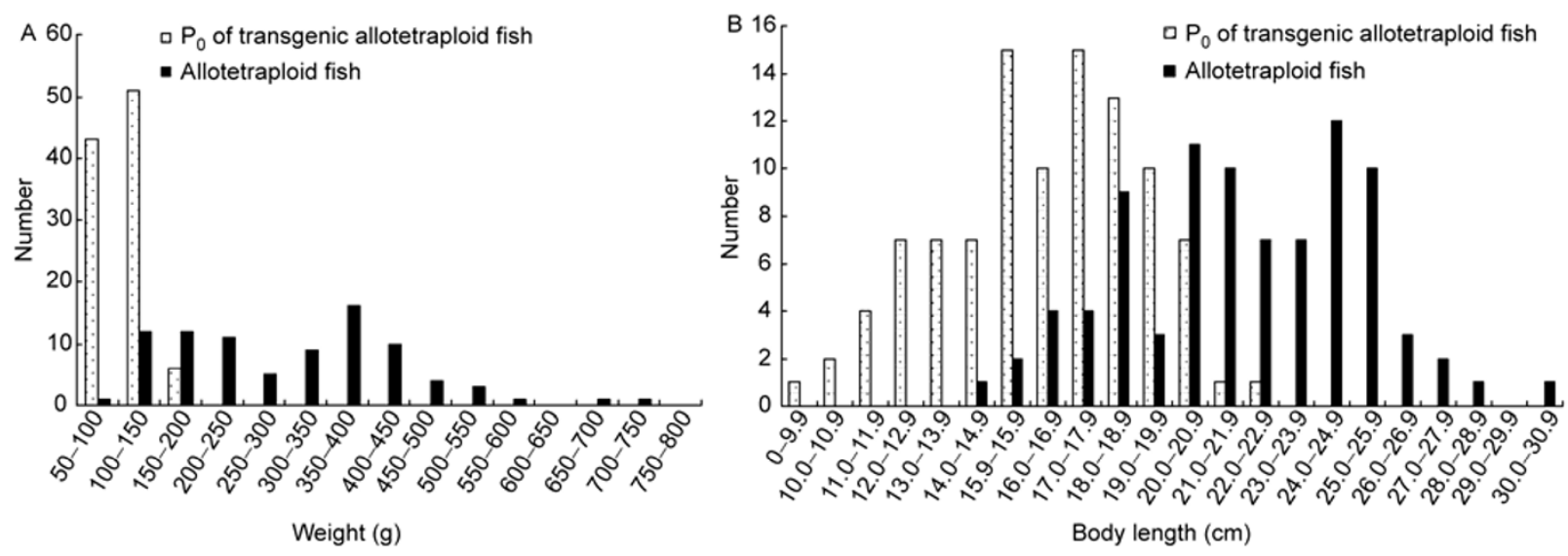

Figure 2 The weight (A) and body length (B) of 150 day-old transgenic allotetraploid fish and control fish. 
Table 1 The weight and body length of 150 day-old $\mathrm{P}_{0}$ transgenic allotetraploid fish and control fish ${ }^{\mathrm{a}}$

\begin{tabular}{|c|c|c|c|c|c|c|}
\hline & \multicolumn{3}{|c|}{ Weight (g) } & \multicolumn{3}{|c|}{ Body length $(\mathrm{cm})$} \\
\hline & Max & Min & Average & Max & Min & Average \\
\hline Transgenic allotetraploid fish & 700 & 70 & $296.3 \pm 70.3$ & 30 & 14.5 & $21.7 \pm 2.4$ \\
\hline Allotetraploid Fish & 195 & 35 & $104.2 \pm 37.4$ & 21.5 & 11.2 & $16.1 \pm 1.8$ \\
\hline Results & \multicolumn{3}{|c|}{ Significant difference, $P<0.01$} & \multicolumn{3}{|c|}{ Significant difference, $P<0.01$} \\
\hline
\end{tabular}

a) $87 \mathrm{P}_{0}$ transgenic allotetraploid fish and 100 control fish were measured.

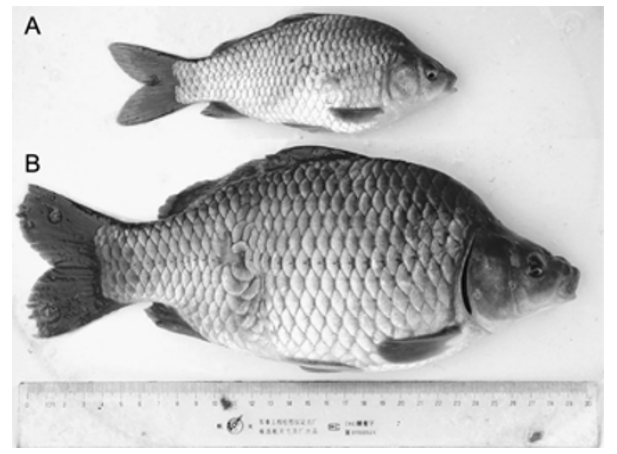

Figure 3 Comparison between 150 day-old $\mathrm{P}_{0}$ transgenic allotetraploid fish and control fish. A, The allotetraploid control fish. B, The black carp $\mathrm{GH}$ gene transgenic allotetraploid fish. The ruler indicates $30 \mathrm{~cm}$.

duced through hybridizing male allotetraploid fish with diploid female crucian carp or female common carp. The establishment of allotetraploid fish species provides an opportunity for producing sterile triploid transgenic fish on a large scale.

Black carp is one of "the Four Chinese Carps". Its good flavor and large size has meant it is an economically important fresh water species. However, there are few reports investigating the molecular biology of black carp. We have already cloned the black carp GH gene cDNA and the promoter of the black carp $\beta$-actin gene. A black carp GH antiserum had been produced and the function of the promoter of black carp $\beta$-actin gene had been studied in vivo $[20,21,23]$. In this paper, the major components of the "all-fish" transgene are from black carp and the data generated in this study showed that the transgene functions well in the host allotetraploid fish.

The contrast cultivation data non-ambiguously showed that the $\mathrm{P}_{0}$ black carp $\mathrm{GH}$ gene transgenic tetraploid fish had a higher growth rate than non-transgenic siblings. Devlin et al. [24] reported that the growth response driven by the $\mathrm{GH}$ transgene is strongly influenced by the intrinsic growth rate and genetic background of the host strain. In hybrids between wild fish and domesticated fish, the growth enhancement due to the inserted $\mathrm{GH}$ transgene was higher than that in domesticated fish [24]. The black carp GH gene transgenic allotetraploid fish grows much faster than the controls (the average weight of transgenic fish was 2.8 times that of the control and the average body length of the transgenic fish was 1.3 times that of the control) and the male parent of the allotetraploid fish, the Xiangjiang River carp, is a kind of wild carp. All these observations are consistent with Devlin's conclusion.

The transgene was detected in the caudal fins from all 20 male transgenic fish; however, the inserted transgene was detected in only 13 of 20 sperm samples from these fish. This might be because of fast egg cleavage and slow integration of the transgene, which is consistent with the mosaicism of the transgene in "the model of transgenic fish" [25].

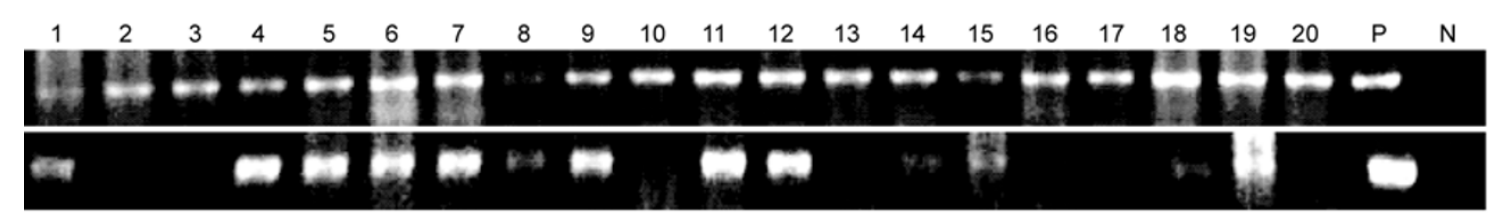

Figure 4 PCR using caudal fin and sperm DNA from $\mathrm{P}_{0}$ transgenic allotetraploid fish (upper, caudal fin; lower, sperm). P, Positive control (pbcAbcGHc); $\mathrm{N}$, negative control (allotetraploid fish); 1-20, individual male $\mathrm{P}_{0}$ transgenic allotetraploid fish.

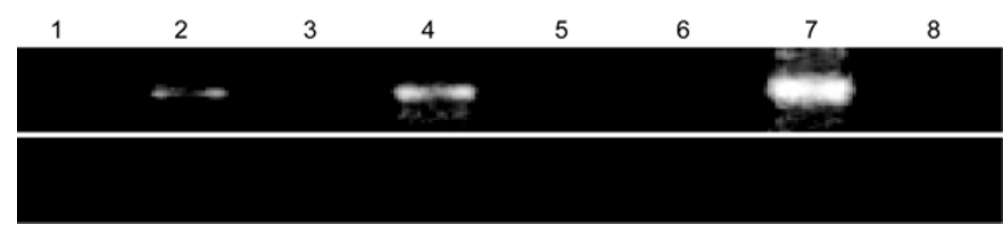

Figure 5 RT-PCR using total RNA from $\mathrm{P}_{0}$ transgenic allotetraploid fish (upper, RT-PCR; lower, NO-RT control). 1, Spleen; 2, muscle; 3 , liver; 4, kidney; 5 , intestine; 6 , heart; 7 , ovary; 8 , fin. 
It is feasible to obtain a pure line of GH transgenic allotetraploid black carp through inbreeding or via a gynogenesis strategy based on the $\mathrm{P}_{0}$ transgenic allotetraploid fish.

Sperm could be pressed out from 32 male 150 day-old transgenic allotetraploid fish but not from the controls. A similar phenotype was reported in grass carp GH transgenic carp [22]. The sexual maturation period of the grass carp $\mathrm{GH}$ transgenic carp is longer than the controls, which is based on a study of the $\mathrm{P}_{0}$ generation and the subsequent five generations of transgenic carp (Zhu ZuoYan, personal communication). All the above data indicate that the influence of the GH transgene on the sexual maturation of the host fish varied with the host ploidy. The mechanism behind why the inserted black carp GH gene shortens the sexual maturation period of allotetraploid fish will be further studied.

A black carp GH gene transgenic allotetraploid fish with an increased growth rate was obtained in this study through the introduction of the "all-fish" transgene, $p b c A b c G H c$, into the eggs of allotetraploid fish, in which the promoter of the black carp $\beta$-actin gene and the ORF of the black carp GH gene are precisely assembled. This study has built a solid foundation for the establishment of a pure line of black carp GH gene transgenic allotetraploid fish, and has provided additional data for molecular biological studies on black carp and allotetraploid fish. Sterile triploid transgenic fish will be generated from the breeding of black carp $\mathrm{GH}$ gene transgenic allotetraploid fish with grass carp GH gene transgenic carp. This strategy will have no ecological safety problem and can service the potentially huge market.

This work was supported by the National Special Fund for Scientific Research in Public Benefits (Grant No. 200903046-08), the National Basic Research Program of China (Grant No. 2007CB109006), the National Natural Science Foundation of China (Grant No. 30500294) and the Excellent Talent Program of Hunan Normal University (Grant No. ET31004).

1 Zhu Z, Li G, He L, et al. Novel gene transfer into fertilized eggs of goldfish (Carassius auratus). J Appl Ichthyol, 1985, 1: 31-34

2 Zeng Z Q, Zhu Z Y. Transgenes in F4 pMThGH-transgenic common carp (Cyprinus carpio L.) are highly polymorphic. Chin Sci Bull, 2001, 46: 143-148

3 Cachot J, Law M, Pottier D, et al. Characterization of toxic effects of sediment-associated organic pollutants using the $\lambda$ transgenic Medaka. Environ Sci Technol, 2007, 41: 7830-7836

4 Fu C, Li D, Hu W, et al. Fast-growing transgenic common carp mounting compensatory growth. J Fish Biol, 2007, 71: 174-185

5 Rosa C E, Fiqueiredo M A, Lanes C F, et al. Metabolic rate and reactive oxygen species production in different genotypes of GH-transgenic zebrafish. Comp Biochem Physiol B Biochem Mol Biol, 2008, 149:
209-214

6 Abad Z, Gonzalez R, Mendoza I, et al. Production of a high percentage of male offspring in growth-enhanced transgenic tilapia using Oreochromis aureus ZZ selected pseudofemales. Aquaculture, 2007, 270: $1-4$

7 Zhu Z Y, Zeng Z Q. Open a door for transgenic fish to market. Biotech inf, 2000, 1: 1-6

8 Reynold M, Maria T H, Amilcar A, et al. Tilapia chromosomal growth hormone gene expression accelerates growth in transgenic zebrafish (Danio rerio). Elctron J Biotechnol, 2001, 4: 1-7

9 Sun X W, Liang L Q, Yan X C, et al. Research on transgenic fish as food. High Tech Lett, 1998, 3: 50-55

10 Wang J L. Transgenic organisms and biosafety. Chin J Ecol, 2006, 25: 314-317

11 Hu W, Wang Y P, Zhu Z Y. Progress in the evaluation of transgenic fish for possible ecological risk and its containment strategies. Sci China Ser C-Life Sci, 2007, 50: 573-579

12 Sundström L F, Lõhmus M, Johnsson J I, et al. Growth hormone transgenic coho salmon pay for growth potential with increased predation mortality. Proc R Soc Lond B, 2004, 271: 350-352

13 Li D L, Fu C Z, Hu W, et al. Rapid growth cost in "all-fish" growth hormone gene transgenic carp: reduced critical swimming speed. Chin Sci Bull, 2007, 52: 1501-1506

14 Sundström L F, Lõhmus M, Devlin R H. Migration and growth potential of coho salmon smolts: implications for ecological impacts from growth-enhanced fish. Ecol Appl, 2010, 20: 1372-1383

15 Sundström L F, Lõhmus M, Tvmchuk W E, et al. Gene-environment interactions influence ecological consequences of transgenic animals. Proc Natl Acad Sci USA, 2007, 104: 3889-3894

16 Jhingan E, Devlin R H, Iwama G K. Disease resistance, stress response and effects of triploidy in growth hormone transgenic coho salmon. J Fish Biol, 2003, 63: 806-823

17 Devlin R H, Sundström L F, Muir W M. Interface of biotechnology and ecology for environmental risk assessments of transgenic fish. Trends Biotechnol, 2006, 24: 89-97

18 Liu S J, Liu Y, Zhou G J, et al. The formation of tetraploid stocks of red crucian carpxcommon carp hybrids as an effect of interspecific hybridization. Aquaculture, 2001, 192: 171-186

19 Liu S J, Hu F, Zhou G J, et al. Gonadal structure of triploid crucian carp produced by crossing allotetraploid hybrids of Carassius auratus red var. (우) $\times$ Cyprinus carpio (ठ) with Japanese crucian carp (Carassius auratus T. ETS). Acta Hydrobiol Sin, 2000, 24: 301-306

20 Feng $\mathrm{H}$, Chen J, Luo J, et al. Cloning of Black Carp $\beta$-actin gene and primarily detecting the function of its promoter region. Acta Genet Sin, 2006, 33: 133-140

21 Feng $\mathrm{H}$, Zeng Z Q, Cheng J, et al. Molecular cloning and sequencing of growth hormone gene for black carp. Acta Laser Biol Sin, 2002, 11: 407-411

22 Feng H, Zeng Z Q, Liu S J, et al. Studies of F1 of transgenic allotraploid hybrids of Carassius auratus red var. (우) $\times$ Cyprinus carpio $\left({ }^{\Uparrow}\right)$. Acta Genet Sin, 2002, 29: 434-437

23 Feng H, Cheng J, Liu Y, et al. In Vitro expression and antibody preparation of black carp (Mylopharyngodon piceus) GH. Hereditas (Beijing), 2005, 27: 729-734

24 Devlin R H, Biagi C A, Yesaki T Y, et al. Growth of domesticated transgenic fish. Nature, 2001, 409: 781-782

25 Wang Y P, Hu W, Wu G, et al. Genetic analysis of "all-fish" growth hormone gene transferred carp (Cyprinus carpio L.) and its F1 generation. Chin Sci Bull, 2001, 46: 1174-1177

Open Access This article is distributed under the terms of the Creative Commons Attribution License which permits any use, distribution, and reproduction in any medium, provided the original author(s) and source are credited. 